\title{
Anatomie meets Phlebologie: Workshop der VOP-AG in Bochum
}

„Nichts ist so gut, dass es nicht noch verbessert werden könnte“. Unter diesem Motto trafen sich vom 18. bis 19.12.2018 in Bochum Vertreter der AG Varizenoperationen (VOP-AG) mit dem renommierten österreichischen Anatom, Prof. Dr. Erich Brenner, Sektion klinisch-funktionelle Anatomie der
Medizinischen Universität Innsbruck. Ziel der Veranstaltung war es, durch den anatomisch - klinischen Austausch eine Optimierung der operativen Gefäßzugänge zu erzielen. Der Focus lag vor allem auf der Vermeidung von Läsionen an den Lymphbahnen. Nach 2 Tagen klinischem und wissenschaft- lichem Austausch ergaben sich eine ganze Reihe von interessanten Fragestellungen für die weitere Kooperation zwischen Anatomie und Phlebologie. Anatomie meets Phlebologie: ein interessantes Format, sicherlich nicht nur für Operateure.
IMPRESSUM

Verantwortlich für Mitteilungen der DGP:

Dr. med. Erika Mendoza 